\title{
EDITORIAL
The use of ICG videoangiography and FLOW 800 analysis
}

\author{
Jacques J. Morcos, MD, FRCS(Eng), FRCS(Ed), and Stephan A. Munich, MD \\ Department of Neurosurgery, University of Miami, Florida
}

I $\mathrm{T}$ is an old neurosurgical adage to state, "The carotid artery is a terrible thing to lose." For all too long, the field of cerebrovascular surgery has indeed been synonymous with arteries. After all, that is where most of the neurosurgical drama, triumphs, and disasters really reside. Veins have been "surgical orphans" until quite recently. It is most fitting that the cerebral venous system be "celebrated" in a dedicated journal issue such as this one. It is also most fitting to include in this issue an article describing the results of careful observation of cerebral venous flow, in health and disease states, through the window of semiquantitation.

What is our relationship to cerebral veins in general? Is it amicable or antagonistic? It could be either. Cerebral veins play a complex role in surgical practice, and the senior author (J.J.M.) has always thought of the neurosurgeon's relationship to them as falling into one of two groupings: friend or foe, with a total of 5 categories.

\section{Veins as the Surgeon's Friend}

1) The preserved vein: This occurs when a vein is not central to the surgical approach, and surgery is successfully carried out with utmost attention to the vein's preservation.

2) The sacrificial vein: This occurs when a vein is an unavoidable obstacle to the completion of surgery, and given the presence of collaterals and venous redundancies, it is sacrificed without consequence, resulting in improved surgical access.

3) The assistant vein: This is the vein that lends itself as an avenue/access to cure disease, often the only way to achieve the cure. Typical examples include using venous channels (endovascularly or microsurgically) to occlude a dural arteriovenous fistula (AVF) and, more recently but rarely, a true nidal arteriovenous malformation (AVM) through targeted endovenous embolization.

\section{Veins as the Surgeon's Foe}

4) The offending vein: Here one can list entities such as venous compressive cranial neuropathies, sinus thrombosis, or stenosis causing pseudotumor cerebri, sigmoid diverticula causing pulsatile tinnitus, sinus pericranii, recurrent cavernomas associated with venous anomalies, and others.

5) The offended vein: These are the instances of postoperative edema, infarction, or hemorrhage related to intentional or unintentional loss of a venous channel during surgery, embolization, or after radiosurgery, in the presence or absence of congenital, suspected or unsuspected venous anomalies.

In the study by Acerbi and colleagues, the authors present their experience using indocyanine green videoangiography (ICG-VA) and FLOW 800 analysis to characterize the venous circulation encountered intraoperatively during procedures for a variety of entities (48 aneurysms, 25 revascularizations, 16 AVMs, 14 AVFs, 8 cavernomas, 15 microvascular decompressions, and 46 tumors). ${ }^{1}$ Using these modalities, the authors classified various forms of venous drainage-arterialized veins, thrombosed veins, fast-draining veins with anterograde flow, slow-draining veins with anterograde flow, and slow-draining veins with retrograde flow. They also employed ICG-VA and FLOW 800 during a temporary clip test, which could be thought of as a "provocative" test-the venous equivalent of the "balloon test occlusion"-to determine if a given vein could be sacrificed. Based on this methodology, the authors found that slow-draining veins experienced flow reversal when clipped (suggesting their tolerance to sacrifice due to the assumed presence of collateral venous circulation), whereas fast-draining veins exhibited stagnation when clipped (suggesting their intolerance to sacrifice due to the assumed absence of collateral venous circulation).

The authors should be commended for their effort to 
scientifically assess an intraoperative decision that is often made according to the personal experience of neurosurgeons, pure anatomy, or simply dogma. While these methods have permitted the safe performance of countless neurosurgical procedures over many decades, they (and the present literature) are remarkably devoid of a scientifically based assessment of which veins can be safely sacrificed. The quantification of venous flow, as presented here, certainly aids in the understanding of the local, perilesional venous anatomy but also, more importantly, the physiology. The ability to obtain this information intraoperatively, rather than only with a preoperative angiogram, adds to the utility of this method.

The value of true intraoperative quantification of arterial flow is well established and has been used for nearly 2 decades. ${ }^{2,3}$ The senior author has used intraoperative measurements of arterial flow (not velocity) since 1999 routinely in cerebrovascular surgery and finds it of immense value in bypass surgery (for ischemia, moyamoya disease, and aneurysms) and aneurysm clipping. The technique is complementary to ICG-VA. The use of FLOW 800 analysis, while not really quantitative, is a move in the right direction and is certainly an added tool in evaluating veins.

It behooves us, though, to analyze the authors' study in detail and highlight its limitations. The group from Milan has had a significant interest in this topic. ${ }^{4-6}$ In spite of this, of the 1972 surgical patients treated over 78 months, the application of ICG-VA/FLOW 800 was deemed useful in only $8.7 \%$ of the cases. Of the 5 groupings they established, 2 categories can be described as "nice to know, but now what?": the arterialized group (41/172) and the thrombosed group (1/172). It is certainly comforting to confirm with FLOW 800 a suspected arterialized vein seen under the microscope, although there is rarely any doubt as to its nature. Occasionally, there could be confusion with an artery, although the addition of FLOW 800 is not really needed to make that determination. The situation is similar for the confirmation of a thrombosed vein.

The interesting distinction is between the "antegrade fast" and "antegrade slow" groups (total 128/172; unclear how many in each). The authors arbitrarily chose an arteriovenous 5-second gap mark as a criterion to distinguish the 2 groups. It is unclear where the validation for this came from. Assuming the validity of this choice is not in question, we wonder how reproducible the transit curve is for the "antegrade slow" veins, given that the authors noted that flow in them is often composed of a multitude of streams, presumably some faster than others. What if the region of interest (ROI) chosen falls on one or the other portion of the streams within the same vein? What is the optimal area to sample? It is important to realize that, after all, the FLOW 800 software methodology is only a semiquantitative methodology, in the sense that it maps the transit time of a dye by tracing the average intensity of fluorescence in arbitrary units, units that vary with method of ICG injection (speed, quantity, proximity to vessel of interest, cardiac output, and so on), illumination of surrounding operating room environment, and other factors. It should come as no surprise that $\mathrm{t}_{1 / 2 \max }$ does not correlate with absolute quantitative vessel flow, or regional cortical perfusion for that matter. ${ }^{7}$
The real purpose of using FLOW 800 is clearly not simply to generate beautiful color-coded vascular maps that augment the microscopic reality, but rather, and as intended by the authors, the goal is to guide intraoperative management of veins. Of the entire patient cohort, only 10 patients had venous sacrifice and then underwent a flow assessment. In none of these patients progression to infarction or hemorrhage occur. The only remaining patients who might have offered an opportunity for hypothesis testing were the 16 patients who underwent a test occlusion of the vein. Unfortunately, here again the opportunity for meaningful conclusions is limited. Even though the authors addressed these limitations in their discussion, it is worth repeating. Of that group of patients, 13 had "slow" veins and 12/13 exhibited venous redistribution with the clip test (that is, a "positive" result, as labeled by the authors) and thus underwent vein sacrifice with no sequelae. The remaining 3 patients had "fast" veins that demonstrated stagnation on temporary clipping (a "negative" result) and did not undergo sacrifice. It is clear that a phenomenon of self-fulfilling prophecy is taking place. In the absence of venous infarctions developing in any of the groups, we are unable to calculate a negative predictive value for the clip test. All one can conclude is that most "slow" veins will "pass" the clip test and will probably tolerate sacrifice. No conclusions can be dawn about the "fast" veins, which were "presumed" to be essential.

We also believe that a small clarification is in order. "Stagnation" in a vein that is being clipped needs to be clarified, as it does not necessarily imply poor collaterals. Stagnation within a vein (sometimes even within a major sinus) is not infrequently observed when tumor invades these structures, yet the vast majority of these patients exhibit no symptoms due to the presence of collateral venous circulation. If a parasagittal bridging vein receiving 2 tributaries is clipped near its entrance into the superior sagittal sinus, and venous flow redistributes such that the actual vein itself becomes stagnant, while one tributary now drains into the other tributary, clearly the conclusion should be that the bridging vein is redundant and will likely tolerate sacrifice, as long as the venous union point is spared. The "stagnation" concept should refer to the entire venous complex serving the tested vein, not just the vein itself. Future studies along these lines should also investigate the change in flow of veins immediately adjacent to the ones being test occluded. How can we get a full understanding of hemodynamic shifts without rising above the trees and looking at the entire forest? Interesting data will likely emerge.

ICG-VA and FLOW 800 are just one piece of analysis necessary in dealing with veins. A thorough understanding of preoperative venous angiographic anatomy remains important. After all, the choice of approach is often dictated by it, well before an opportunity to perform intraoperative ICG has arisen. A presigmoid petrosal approach may not even be considered if the superior petrosal sinus is dominant or the Labbé complex in intratentorial. The distinction between pathological states and physiological states is critical: sacrificing a petrosal vein in the setting of a petroclival meningioma is clearly more consequential than doing this in the setting of a microvascular decom- 
pression for trigeminal neuralgia, where none of the other veins are compromised. And lastly, it is often forgotten that a vein may look, at first glance, impossible to spare during initial dissection, and yet it is remarkable how further meticulous and patient dissection of arachnoidal bands can liberate the vein enough to allow the completion of surgery without venous sacrifice.

This article should entice all readers to think quantitatively about blood flow in general and venous flow in particular. The use of ICG-VA/FLOW 800 does not answer fully the quantification quest, but it is certainly a step in the right direction. As long as its intrinsic limitations are appreciated, it will improve our understanding of hemodynamics, until more advanced technologies emerge that would much more precisely measure venous flow, in health and disease states, as well as model the consequences of venous manipulation and sacrifice.

https://thejns.org/doi/abs/10.3171/2018.4.FOCUS18207

\section{References}

1. Acerbi F, Vetrano IG, Sattin T, Falco J, de Laurentis C, Zattra CM, et al: Use of ICG videoangiography and Flow 800 analysis to identify the patient-specific venous circulation and predict the effect of venous sacrifice: a retrospective study of 172 patients. Neurosurg Focus 45(1):E7, 2018

2. Amin-Hanjani S, Meglio G, Gatto R, Bauer A, Charbel FT:
The utility of intraoperative blood flow measurement during aneurysm surgery using an ultrasonic perivascular flow probe. Neurosurgery 62:1346-1353, 2008

3. Fagundes-Pereyra WJ, Hoffman WE, Misra M, Charbel FT: Clip readjustment in aneurysm surgery after flow evaluation using the ultrasonic perivascular probe: case report. Arq Neuropsiquiatr 63:339-344, 2005

4. Ferroli P, Acerbi F, Tringali G, Albanese E, Broggi M, Franzini A, et al: Venous sacrifice in neurosurgery: new insights from venous indocyanine green videoangiography. J Neurosurg 115:18-23, 2011

5. Ferroli P, Nakaji P, Acerbi F, Albanese E, Broggi G: Indocyanine green (ICG) temporary clipping test to assess collateral circulation before venous sacrifice. World Neurosurg 75:122-125, 2011

6. Ferroli P, Tringali G, Albanese E, Broggi G: Developmental venous anomaly of petrous veins: intraoperative findings and indocyanine green video angiographic study. Neurosurgery 62:ONS418-ONS421, 2008

7. Prinz V, Hecht N, Kato N, Vajkoczy P: FLOW 800 allows visualization of hemodynamic changes after extracranial-tointracranial bypass surgery but not assessment of quantitative perfusion or flow. Neurosurgery 10 (Suppl 2):231-239, 2014

\section{Disclosures}

J.J.M. reports owning stock in Kogent. 